\title{
Engineering the SDL Formal Language Definition
}

\author{
Andreas Prinz ${ }^{1}$ and Martin v. Löwis ${ }^{2}$ \\ ${ }^{1}$ DResearch Digital Media Systems GmbH, Otto-Schmirgal-Str. 3, \\ D-10319 Berlin \\ prinz@dresearch.de \\ ${ }^{2}$ Hasso-Plattner-Institut für Softwaresystemtechnik GmbH, Postfach 900460, \\ D-14440 Potsdam \\ martin.vonloewis@hpi.uni-potsdam.de
}

\begin{abstract}
With the latest revision of the ITU-T Specification and Description Language (SDL-2000), a formal language definition based on the concept of Abstract State Machines (ASMs) became integral part of the standard. Together with the formal definition, we have developed software tools that allow executing the formal language definition on a computer. In doing so, we found that tools greatly help to eliminate numerous errors from the formal definition, which likely would have not been found without tools.
\end{abstract}

\section{Introduction}

Defining the formal semantics of programming languages is an area of ongoing research. While formalizing the syntax of the language by means of grammars is wellunderstood, various techniques have been studied to define other properties of the language. We think that a language is sufficiently formalized if the following aspects are defined [1]:

1. The lexis: How to transform the input sequence of bytes into a sequence of tokens?

2. The syntax: Given a sequence of tokens, is that a sentence of the language?

3. The well-formedness rules (static semantics): Given a sentence of the language, does that meet certain additional constraints?

4. The run-time (or dynamic) semantics: What does the program do when executed?

In this paper, we discuss these aspects of the language definition for the ITU-T Specification and Description Language [2]. We first introduce the language SDL, and continue with an overview of the formal semantics, covering each of the four aspects.

In our experience, defining a formal semantics for a language is not useful without sufficient tool support. Tools should be used to verify that the formal definition is consistent in itself, and to validate it agrees with the intended semantics of the language. Unfortunately, no single tool can be used to automatically process a formal language definition from the version that is published as an international standard. Consequently, we have developed our own tool chain (building on existing tools where possible) to process the formal semantics definition of SDL. The tools and the results we achieved by using the tools are presented in subsequent sections. We hope to expose this approach to a wider audience, and to encourage researchers to copy either the general approach or the choice of specific tools for their language of interest.

E. Najm, U. Nestmann, and P. Stevens (Eds.): FMOODS 2003, LNCS 2884, pp. 47-63, 2003.

(C) IFIP International Federation for Information Processing 2003 
The SDL formal semantics was developed by an international expert team including the authors of this paper ${ }^{1}$. Due to this effort SDL-2000 does now have a complete semantics for the full language, not only for a subset of it. This semantics is precise for all aspects of the language including syntax and semantics.

Success of the development of a formal semantics for a programming language depends on many aspects, of which this paper covers only a few. In particular, we believe that standardization of the formal language definition is quite important: If the formal language definition is the official semantics of the language, practitioners will use the formal semantics for their own work. For SDL-2000, we managed to convince the SDL standards body (ITU-T Study Group 10) to accept the formal semantics presented in this paper as the official semantics definition of SDL.

\section{The Language SDL-2000}

SDL (Specification and Description Language) is a language for the description of reactive systems [2]. Although developed for the telecom industry, SDL is used in other areas of the industry, as well. In the area of telecommunications, SDL can be used for the following application fields: call and connection processing, maintenance and fault treatment, system control, operation and maintenance functions, network management, data communication protocols, and telecommunication services ${ }^{2}$.

More generally, SDL can be used for the functional description of all systems that can be described with a discrete model; that is where the object communicates with its environment by discrete messages [2]. Core concepts to describe such systems are:

- agents, which are used to describe active objects which respond to external stimuli;

- channels, which connect agents;

- signals, which are transmitted via channels;

- data, which describe the state of agents and the parameters of signals.

SDL features both a graphical and a textual syntax. While the graphical syntax allows human readers to grasp the structure of the system and the behaviour of an agent more easily, a machine more easily processes the textual syntax.

\section{The SDL Formal Semantics}

In November 2000, the formal semantics of SDL-2000 was officially approved to become part of the SDL language definition [3]. Currently, it is the only comprehensive and complete formal semantics of SDL-2000, covering all static and dynamic

1 It should be noted that the SDL formal semantics was developed in only one years time, which is really fast taking into account the size of the informal language description (without examples and indexes) taking 350 pages.

2 SDL is a language with a long tradition. It was first defined in 1976 and evolved since then to a very complex language. It is standardised by the International Telecommunication Union (ITU-T) by the standard Z.100. Since 1988 there is a formal semantics standardised together with the (informal) language definition. The SDL-2000 formal semantics was written in the ASM formalism ([1], [6]) in contrast to the 1988 formalisations written in Meta IV and CCS. 
language aspects. From the beginning it was agreed to define the SDL formal semantics to be truly executable. In this section, the static and dynamic semantics of SDL2000 are surveyed. Further details on the dynamic semantics and, in particular, on the use of the ASM formalism can be found in [6].

\subsection{Static Semantics}

The static semantics covers transformations and checks that can be done before executing a specification. In the scope of SDL, there are two major parts of the static semantics:

- Well-formedness conditions: As usual, the SDL concrete syntax is given in a context-free way. Additional constraints are imposed using context conditions.

- Transformations: In order to cope with the complexity of the language SDL, the standard Z.100 identifies certain concepts to be core concepts and defines transformations of various other concepts into these core concepts.

Starting point for defining the static semantics of SDL is a syntactically correct SDL specification as determined by the SDL grammar. In Z.100, a concrete textual, a concrete graphical, and an abstract grammar are defined using Backus-Naur-Form (BNF) with extensions to capture the graphical language constructs. From a syntactically correct SDL specification, an abstract syntax tree (AST) is derived by standard compiler techniques (namely, parser construction for a context-free grammar). The structure of this AST is defined such that it resembles the concrete textual and the concrete graphical grammars. The correspondence between the concrete grammars and a first abstract syntax form, called ASO, is almost one-to-one, and removes irrelevant details such as separators and other 'purely' lexical information. A second step translating AS0 to the final abstract syntax form, called AS1, is formally captured by a set of transformation rules together with a mapping. This results in the following structure of the formalization (see figure 1):

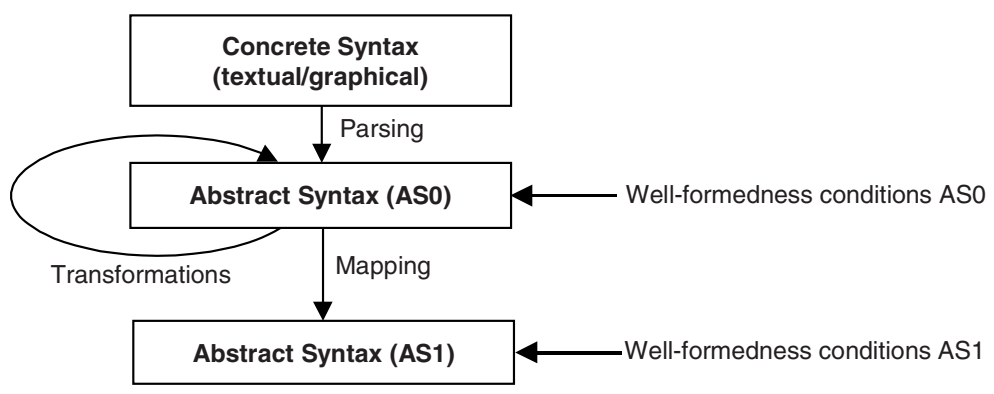

Fig. 1. Overview of the static semantics

- Transformation rules modifying AS0 trees are described in so-called model paragraphs of Z.100, and are formally expressed as rewrite rules.

- After application of the transformations, the structure of the AS0 tree is similar to an AS1 tree. This means that the mapping from AS0 to AS1 is almost one-to-one.

- The well-formedness conditions are split into conditions on AS0 and AS1 (see figure 1). They are formalized in terms of first-order predicate calculus. 
Transformations. Z.100 prescribes the transformation of SDL specifications by a sequence of transformation steps. Each transformation step consists of a set of single transformations as stated in the Model sections of Z.100, and determines how to handle one special class of shorthand notations, i.e., abbreviations. The result of one step is used as input for the next step. The following excerpt of Z.100 prescribes how to deal with a particular shorthand notation, namely a list of output signals:

If several <signal identifier $>$ s are specified in an $<$ output body $>$, this is derived syntax for specifying a sequence of $<$ output $>\mathrm{s}$ in the same order as specified in the original <output body $>$.

The rule states that a list of output signals is a shorthand (called "derived syntax") for a sequence of single outputs.

To formalize the transformation rules of Z.100, rewrite rules are used. A single transformation is realized by the application of a rewrite rule to the specification, which essentially means to replace parts of the specification by other parts as defined by the rule. The following rewrite rule formalizes the transformation above:

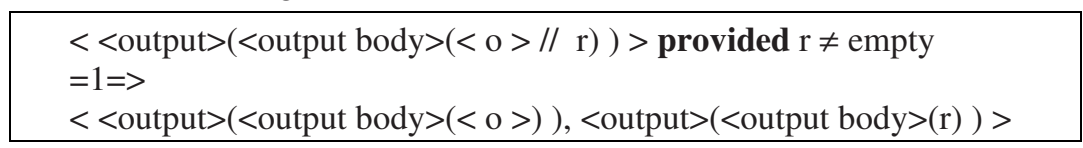

The left hand side (above the arrow) gives the application context of the rule, i.e. an output body containing more than one <signal identifier $>$, in the abstract syntax tree. The right hand side defines how this part of the ASO tree is to be transformed. The number in the operator $=n=>$ indicates the sequence of transformation steps. All the rules are applied as long as they are enabled. For instance, if the sequence of signal identifiers has 3 elements, two applications of the previous rule are required.

Well-Formedness Conditions. The well-formedness conditions define additional constraints that a specification has to satisfy. These constraints cannot be expressed in $\mathrm{BNF}$, though they are static and can be defined and checked independently of the dynamic semantics definition. An SDL specification is valid if and only if it satisfies the syntactical rules and the static conditions of SDL.

Here is a sample resolution rule taken from Z.100, and its formalization:

The $<$ signal identifier $>$ in an <output body $>$ must denote a $<$ signal definition $>$.

$\forall$ o $\in<$ output body $>$ : o.s-<signal identifier $>$.refersto s $_{0} \in<$ signal definition $>$

There is again an application context for this rule given after the $\forall$ symbol - the rule is applicable for <output body $>$ AS0 nodes. The <signal identifier $>$ selected from this node must refer to a <signal definition $>$. The actual resolution is done by the function refersto ${ }_{0}$, which is formally defined in [3] covering about four pages.

\subsection{Dynamic Semantics}

The dynamic semantics defines the dynamic properties resulting from the execution of valid SDL specifications, i.e., their legal behavior. It is based on Abstract State Machines (ASMs) introduced by Yuri Gurevich [5], and defines, for each SDL specifica- 
tion, a distributed real-time ASM, covering concurrency, asynchronicity, and time (see figure 2$)^{3}$.

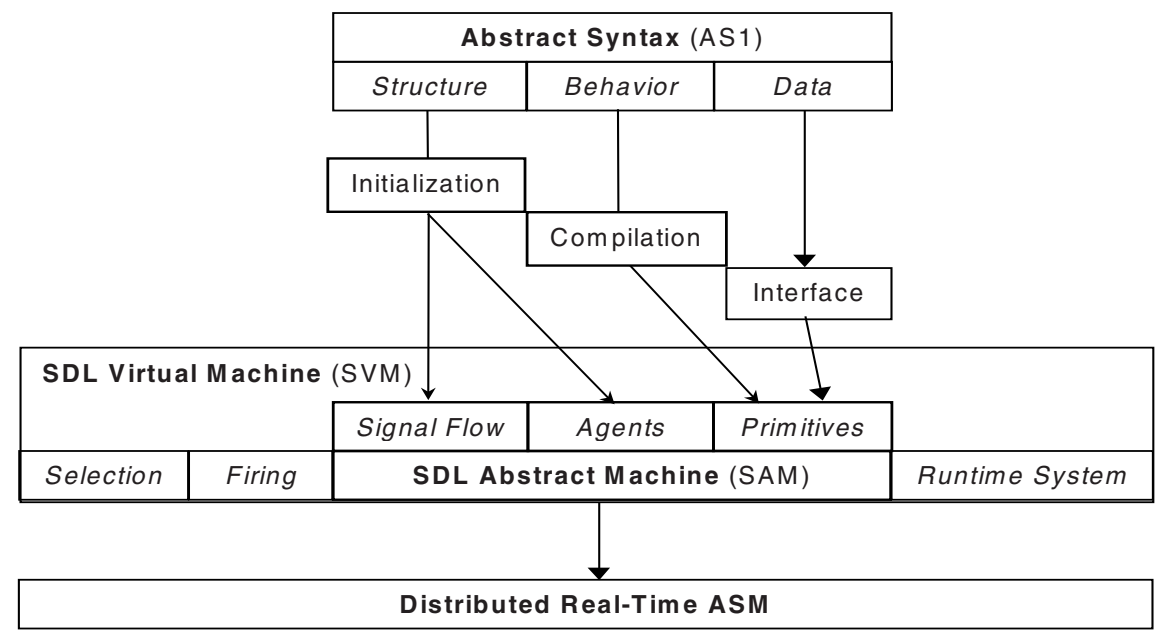

Fig. 2. Overview of the dynamic semantics

The core of our model is a logical hardware called SDL Abstract Machine (SAM), providing a dynamic architecture according to the SDL specification under execution, various types of agents, and an instruction set. Each SDL specification is mapped by the formal semantics to the SAM. The execution of the specification is then performed within the SAM using an abstract operating system called SVM (SDL virtual machine).

SDL Virtual Machine. The SDL Virtual Machine, or SVM, has the SDL Abstract Machine, or SAM, as its heart. Essentially, the SVM adds a run-time system to the SAM, which consists of the programs to drive the execution of the behavior primitives $^{4}$, and the run-time libraries used within the behavior primitives. The SVM consists of fixed parts, which are independent of the actual SDL specification, and dynamic parts created by traversing the abstract syntax ${ }^{5}$. The SAM consists of the following parts (see figure 2):

- Signal Flow Model, which defines a uniform treatment of signal flow related aspects, in particular, asynchronous communication between agents through exchange of signals via channels connected to gates,

- SAM Agents, which model the SDL concepts 'SDL agent', 'SDL agents set', and 'SDL channel', and

- Behavior Primitives, which can be seen as the instructions of the SAM.

3 This means the SDL dynamic semantics is an operational one which is based on state transitions in terms of abstract state machines. Please note that no inference or rule system is involved in this kind of semantics definition (as opposed to a denotational semantics).

4 the selection and firing code.

5 both in the process of behavior compilation, and by recursively unfolding the system structure. 
The signal flow model is based on a decentralized control mechanism for defining asynchronous communication between SDL agents (such as processes, blocks or a system). Basically, it deals with the transportation of signals between (SDL) agents via their gates. Exchange of signals between SDL agents and their environment is modeled by means of gates from a static domain GATE. A gate forms an interface for serial and unidirectional communication between two or more agents. Accordingly, gates are either classified as input gates or output gates.

A static domain SIGNAL represents the set of all signal types as declared by an SDL specification. Dynamically created signal instances belong to a dynamic domain SIGNALINST. Basic functions on signals are signalSender, toArg, and viaArg yielding the sender process, the destination, and optional constraints on admissible communication paths, respectively.

SAM Agents. A distributed ASM defines an asynchronous computation model consisting of some finite collection of autonomously operating agents. Each agent executes a program, where agents that execute the same program are considered to be of the same type. Even SDL connections are modeled as agents executing the program LINKPROGRAM ${ }^{6}$. Agents interact with each other by reading and writing shared locations of global machine states. In the case of the SDL semantics these shared locations are the gates. The underlying semantic model resolves potential conflicts by making nondeterministic choices according to the definition of partially ordered machine runs [5].

The behavior of a link is stated by the state transition rule FORWARDSIGNAL forming the program LINKPROGRAM. For improved readability of ASM programs, complex transition rules are structured by means of rule macros that often have formal parameters. In the definition shown below of the rule ForWARDSIGNAL, Self identifies a particular link agent (the one executing the rule).

A link agent $l$ basically performs a single operation, namely: signals received at gate l.from are forwarded to gate l.to. Whenever $l$ is applicable to a waiting signal si (as identified by the l.from.queue.head), it removes si from l.from.queue and inserts it into l.to.schedule. Competing attempts of two or more link agents to forward the same signal si cannot cause a duplication of the signal. Technically, this property is ensured by the underlying concurrency model (cf. the coherence condition in the definition of partially ordered runs [5]).

FORWARDSIGNAL $\equiv$

if Self.from.queue $\neq$ empty then

let $s i=$ Self.from.queue.head in

if Applicable(si.signalType,si.toArg,si.viaArg,Self.from,Self) then

DELETE(si,Self.from)

INSERT(si,now +Self.delay, Self.to)

\footnotetext{
${ }^{6}$ Note that the resulting signal flow model architecture is fairly robust allowing for the incorporation of additional features in future versions of SDL. For instance, one may have channels with more complex properties (like unreliable transmission behavior) and a dynamically changing communication infrastructure (with channels being added and removed at run time). Such extensions can be easily expressed on the basis of the decentralized signal flow model without any major revision of the current definitions.
} 


\section{si.viaArg := si.viaArg \\{ Self.from.nodeAS1.node.AS1ToId, Self.nodeAS1.node.AS1ToId\}
endif
endlet
endif

Links are just a special case of agents connected via gates. In the SDL formal semantics, there are also process set agents and process agents. The behaviour of process set agents is similar to the link behaviour. The behaviour of process agents is given by the SDL specification and transformed to behaviour primitives as described below.

Behaviour Primitives. The behaviour described in the SDL specification is transformed by the formal semantics to a set of behaviour primitives, forming the machine code of the SAM. This transformation is formalised using a compilation function. The primitives are fairly high-level and correspond to the possible SDL actions. This way, the formal semantics is working on abstract code at runtime and not on the abstract syntax tree.

\section{The Development Process of the Formal Language Definition}

The SDL language specification consists of several parts. Some of these are primarily informal, and given in plain English; other parts are primarily written in formal languages:

- The main part of the language definition in the ITU recommendation Z.100 [2] defines the syntax of SDL, by providing an EBNF (extended Backus-Naur-Form) grammar; it defines the semantics of SDL using English text.

- Annex A is the index of the non-terminal symbols of the grammar.

- Annex D defines the predefined data (package predefined) using a formal syntax. The semantics of the predefined data is given informally in this annex.

- Annex F [3] defines the formal semantics. This annex consists of three parts: F.1 (overview of the formal semantics), F.2 (static semantics) and F.3 (dynamic semantics).

Different working groups developed these documents over a period of several years. They were working on the informal and the formal definition in parallel. In order to reach a consistent language definition, a development process was followed to systematically formalise the completed parts of the informal definition.

Due to the groups involved, and the documents produced, the development process had several phases, as shown in figure 3 .

\section{The Tool Chain}

Two main approaches lead to the discovery of inconsistencies and other problems in the language definition. On the one hand, the mere attempt to formalise a certain con- 
cept lead to the observation, that certain constellations have no clear meaning. In other words, we found some errors by "just looking" at the specification?.

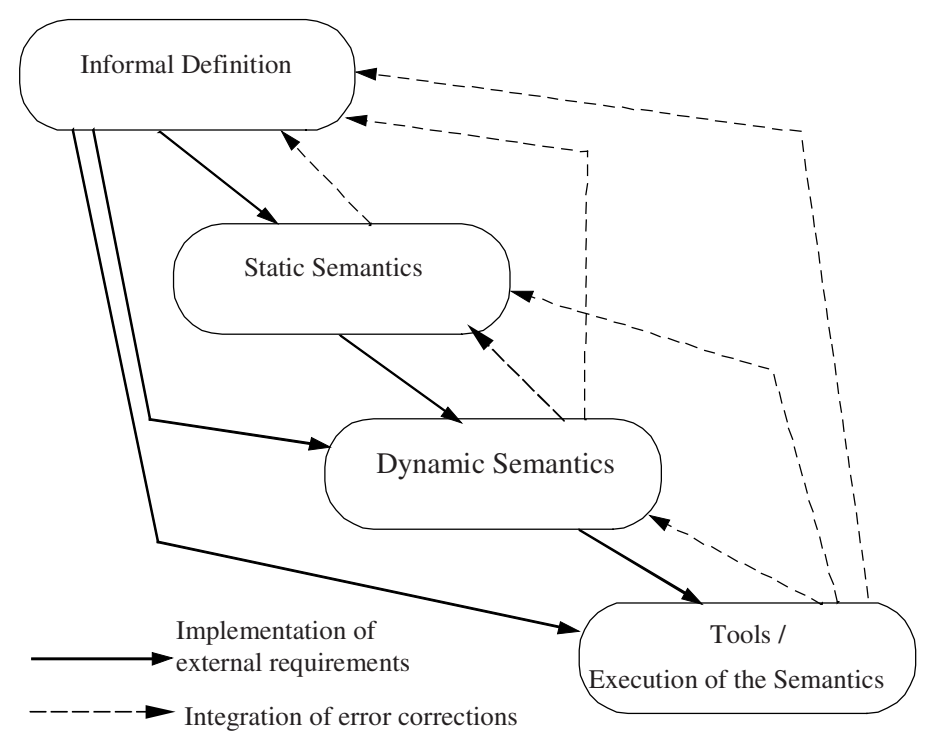

Fig. 3. Development process of the formal semantics definition

On the other hand, various problems where detected using software tools. Figure 4 gives an overview of these tools. The main idea of the tools is to use the formal semantics as a high-level description of an SDL compiler, which is turned into a lowerlevel description using a transformation tools called SDLC. However, also the lowerlevel description is still on a high level such that the transformation does not need to introduce major changes to the description ${ }^{8}$.

As required by the ITU-T administration, the formal semantics documents had to be maintained using the Microsoft Word [9] tool. To define the formal semantics, the following features of that tool were used:

- Using text labels and hypertext links, easy navigation in the document becomes possible. For example, every call to a function refers (as a hyperlink) to the function definition.

7 Even though this approach is hardly systematic and trustworthy, it is quite reliable if executed carefully: If, for example, the language designer finds that the formalisation of an algorithm requires data that are unavailable to that algorithm, the language designer will try to solve this by extending the formal language definition with the necessary data. In turn, he might find that this extension cannot be implemented, and that the informal definition needs to specify a different algorithm.

8 Please note that there are two transformations to be considered. The first transformation is from the formal semantics definition of SDL to the SDL reference compiler, shown in the left part of figure 4. The second transformation is from a concrete SDL specification to an output trace of this specification, which is shown in the right part of the figure. 
- Using auto correction entries, entering these links is simplified. For example, entering the string Igdecision results in a hyperlink to the grammar rule Decision.

- Using Visual Basic macros, entering the auto correction entries is simplified. One such macro searches the entire document for grammar rules, function definitions, and other definitions, and creates the necessary auto correction entries.

- Using character and paragraph formats, both the generator for the auto correction entries, and the extraction process is driven.
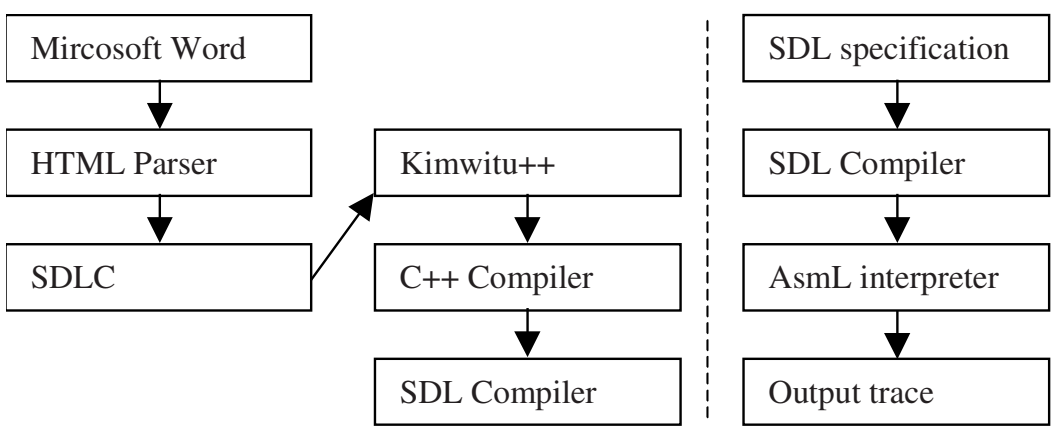

Fig. 4. The Tool Chain

The Word document has, alternating, formal and informal parts. The informal parts describe the desired operation of the formulae. Although very important to the reading human, they are not needed to execute the formal language definition. The formal parts are not easy to process as long as they are stored in the internal Microsoft Word format. To allow further processing of the formal definition, it is first converted into HTML using the HTML generator built into Word 2000, which is then processed with an HTML parser to extract the formulae.

Even though it may appear that the chosen approach of transformation steps is tied to the specific input format (i.e. Microsoft Word), it would be possible to adapt the process to different input formats. For example, if the input was available as a PostScript file, the HTML parser would need to be replaced with a PostScript parser.

These extracted files are then compiled using the compiler SDLC (developed in parallel to the language definition) into Kimwitu++ code [4, 8]. Kimwitu++ compiles the code into $\mathrm{C}++$ code. A $\mathrm{C}++$ compiler creates then an executable program from the $\mathrm{C}++$ code.

The resulting executable program is an SDL reference compiler automatically derived from the language definition. It takes an SDL specification as its input and produces a set of AsmL [7] fragments. Those are combined with other AsmL fragments (extracted from the Word document). The AsmL compiler translates all these fragments into a $\mathrm{C \#}$ program, which the $\mathrm{C \#}$ compiler [10] compiles into a Microsoft .NET executable program.

Executing this program produces a possible trace of the original SDL specification.

Nearly all of these translation steps can fail. We will demonstrate a few typical problems found using the tools, and how they can be corrected. 


\section{Errors Detected by SDLC}

If the SDLC compiler detects errors, they indicate problems in the formulae extracted from the word document. Several such errors are shown in the following sections.

\subsection{Bugs in the Extraction Tool}

When processing the HTML generated with Microsoft Word, specific aspects of that HTML format had to be considered, in particular mathematical characters: The formulae of the SDL language definition make use of special characters (such as $\exists$ and $\forall$ ). These are represented using the symbol font in HTML, whereas the extracted form expected by SDLC uses an ASCII notation (lexists and Iforall) for these characters. Each such character has to be specifically added to the extraction tool. It took some time to enable the extraction tool to correctly extract the formal texts.

\subsection{Formatting Errors in the Word Document}

The compiler SDLC uses different namespaces for functions, domains, ASM rules, and ASM variables. Those need to be marked up in the Word document with a certain character format, and are converted to prefixes (f-, d-, r-, and a-) during extraction. The extractor produces those prefixes every time the $<$ span $>$ element is encountered in the HTML file. Using this strategy, a number of problems have been found:

- Incorrect mark-up: Sometimes, not only the identifier was marked, but also the white space around it. The extraction tool would then put the prefix in front of the white space, which caused SDLC to report a syntax error. This error needs to be corrected by removing the formatting of the white space.

- Missing mark-up: Some identifiers where not appropriately marked, in particular, if entering the proper mark-up was too tedious for the author of the formal semantics. In those cases, the proper mark-up needed to be added during revision.

\subsection{Syntax Errors}

Passing the extracted files to SDLC for the first time, numerous syntax errors were reported which were not just the result of incorrect formatting. Those errors can be classified in the following categories:

- Systematic usage of incorrect syntax: A number of errors happened as authors of the formal semantics were not always aware of the syntax to be used in the formulae. Using the formulae language just from memory, they naturally made errors in applying the syntax properly. One example is the usage of the case expression. A proper usage example of this expression reads

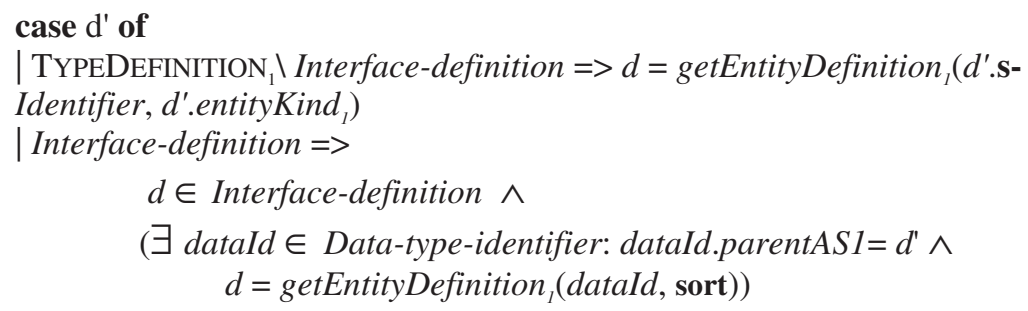




\section{| Syntype-definition $=>$ \\ otherwise False \\ isDirectSuperType $_{1}\left(d, d^{\prime}\right.$.derivedDataType $)$ \\ endcase}

In some cases, this syntax was misspelled: Instead of using of as the keyword, the word in was used, and instead of using "=>" as the separator between the pattern and the result expression, the separator ":" was used. Those errors were detected when passing the input to SDLC, and had to be corrected in the Word document.

- Mismatching parentheses: A large number of missing closing parentheses were found; there were also closing parentheses without a matching opening parenthesis. As the formulae language has several different parentheses kinds ("()", "\{\}", and "<>"), some of these errors resulted from incorrectly swapping closing parentheses.

- Unsupported constructs: A number of syntax errors were caused by using a syntax in a formula which was not supported in SDLC. We could eliminate some of those errors by enhancing SDLC (and thus enhancing the language for formulae). In other cases, the formula had to be rewritten to use only supported constructs ${ }^{9}$.

\subsection{Errors in the SDLC Output}

After successfully processing the formal definition with SDLC producing Kimwitu++ code, we found that the resulting Kimwitu++ code was incorrect, and rejected either by Kimwitu++, or by the $\mathrm{C}++$ compiler. Those errors can be classified as follows:

- Type errors originating from SDLC: In a number of cases, the resulting $\mathrm{C}++$ code showed type errors, even though the formal SDL definition was correct. Some of these errors resulted from an incomplete type analysis inside SDLC: SDLC tries to translate the formulae on a syntactic level only, without performing a complete static analysis. This turned out to be insufficient in some cases, e.g. when the compiler needs to declare the type of a variable in $\mathrm{C}++$, when no such declaration is necessary in the formula. SDLC then uses a heuristics to output a $\mathrm{C}++$ type name; this heuristics turned out to be incorrect. We have then corrected the generator to improve the heuristics, using automatic type conversion available in $\mathrm{C}++$.

- Incorrect arguments to function calls: In the static semantics of SDL, a specification is represented as a tree of a certain grammar. In order to manipulate the tree, new nodes in that tree have to be created, which is expressed as a function call of the non-terminal to be created; the arguments to the function call are the subtrees appearing on the right-hand side of the grammar production. These calls are nearly-literally copied into the $\mathrm{C}++$ code, so that the $\mathrm{C}++$ compiler would verify the correct usage of the non-terminal. In a number of cases, there were arguments missing, or extra arguments given. We found that in most of these cases, the error resulted from a change to the grammar that was inconsistently applied (i.e. ignoring all occurrences of the non-terminal that was changed).

9 For example, to denote a sequence domain with elements originating from a domain $D$, the construct $D^{*}$ is used. To denote an optional value (i.e. a domain that also includes the value undefined), [D] is used. Even though SDLC separates both constructs, it rejects the construct $[D]^{*}$, i.e. a list of optional values. We enhanced SDLC to support this notation as well. 


\section{Executing the Static Semantics}

After correcting the errors described in section 6, a compiler translating SDL to AsmL can be generated and an SDL specification can be passed to the generated reference compiler. This, in turn, can produce various errors, which are shown in the following.

\subsection{Checking the Well-Formedness Conditions}

As the first step in processing an SDL specification, a number of well-formedness conditions (expressed in the predicate calculus) are checked for the input. These checks can fail for a number of reasons:

- Errors in the SDL specification: In the ideal case, a failed condition indicates a true error in the SDL specification. In that case, further processing of the SDL specification is meaningless, and the specification needs to be revised.

- Errors in formalising the well-formedness condition: It is possible that the SDL specification is correct, and that the informal language definition says it is correct, but an error was made when formalising the condition. In that case, the formula of the condition needs to be corrected.

- Errors in the informal definition: A well-formedness predicate may fail if the informal language definition is erroneous or too strong. In this case, the language committee experts need to determine what the intended condition was, and find proper language to describe it. Afterwards, the formalisation needs to be updated.

In addition to conditions that fail, it is possible that a condition erroneously holds, or that its computation does not terminate. The former case is difficult to detect: One needs an SDL specification that is known to be ill-formed, and then find that this specification is not rejected in the reference compiler. Once the problem is detected, it may, again, either need to be fixed in just the formal language definition, or in the informal one as well.

On the other hand, finding a condition that does not terminate is relatively easy: If the compilation of a specification in the reference compiler does not terminate in a reasonable time, the likely cause is an error in some formula. For many constructs, it is obvious that they terminate if all their arguments terminate. We found only two cases of infinite algorithms in the formal semantics, namely infinite recursion ${ }^{10}$ and quantifications over infinite domains ${ }^{11}$.

\subsection{Execution of Transformation Rules}

In SDL, a number of constructs are defined as short cuts for other constructs. They don't have a dynamic semantics of their own, but receive their semantics by means of

\footnotetext{
${ }^{10}$ In order to compute some data, auxiliary functions were used that ultimately invoked the same functions that called them, passing the same arguments that were currently under computation. In these cases, a different algorithm had to be found.

${ }^{11}$ Using quantifications is common to specify well-formedness conditions. Mostly it is obvious that the computation of them will terminate, for instance many of the quantifications run over syntax nodes, and in any specification, there is only a finite number of syntax nodes. However, we found quantifications over the domain of natural numbers, which had to be rewritten to operate only on a finite subset of all numbers.
} 
transformation to other constructs. Those transformations are formalized by means of a rewrite system (executed by an abstract state machine) [1] $]^{12}$. In executing the transformations, we found problems with infinite loops ${ }^{13}$ and skipped transformations ${ }^{14}$ :

\subsection{Generating AsmL}

Once transformations are complete, the resulting syntax tree was compiled into a sequence of AsmL statements, which were then passed to the AsmL compiler. This compiler detected further errors, which can be classified as follows:

- Constructs unsupported in AsmL: The initial release of AsmL did not support a number of constructs needed for executing SDL specifications. In turn, we forbeared usage of AsmL version 1.5, and switched to a beta release of AsmL version 2, which does support the constructs we need.

- Type errors detected by AsmL: The AsmL compiler reported a number of type errors. They mainly resulted from AsmL using a slightly different typing system than the SDL formal semantics, especially as far as undefined is concerned. Fortunately, only a few formulae made use of this typing, and we could eliminate those with additional type declarations in a few places.

- Bugs in AsmL: The initial beta release of AsmL would crash on the generated ASM. Together with the authors of AsmL, we could eliminate these problems.

- Inconsistencies in the semantics definition: Some inconsistencies show up as inconsistent update sets within AsmL. This means that one location gets two different values assigned. This rare kind of errors was easy to correct.

- Ambiguities in the semantics definition: Sometimes there were competing update sets within AsmL. Some of them relate to nondeterminism within SDL (e.g. channels competing for a signal, which could be relayed via different paths). However, some ambiguities related to weaknesses in the formal semantics.

\section{Executing the Dynamic Semantics}

The AsmL compiler (in the .NET revision) takes the generated AsmL input, together with a few runtime functions, and produces a $\mathrm{C} \#$ program. The $\mathrm{C \#}$ compiler then generates a .NET executable, which can be run using the .NET runtime environment.

${ }^{12}$ Each rewrite rule is defined as a pattern over a subtree of the syntax tree which needs to match for the rule to fire, and a result term which determines the syntax subtree that is used as a replacement for the original subtree.

${ }^{13}$ Some of the rewrite rules were applied over and over again. Those rules produced a result tree that matched its own pattern. In some cases, the tree would grow in size each time the transformation was applied, in other cases, it stayed unmodified. In all cases, the formalization was clearly in error. In some cases, also the informal language definition needed to be changed, if it did not deal properly with recursive application of transformation rules.

${ }^{14}$ Some transformations were skipped erroneously, as their patterns did not match (even though they should have). Those errors were typically detected in a later phase of transformations, as the input was then not in the form that those later transformations expected. Various strategies to correct these problems had to be applied. For example, if transformations relied on the output of later transformations as a precondition for their own input, the order of transformations had to be changed. 
Running this program eventually produces an execution trace, which then can be studied to analyze the formal meaning of the SDL specification originally taken as input. For example, when taking the SDL specification

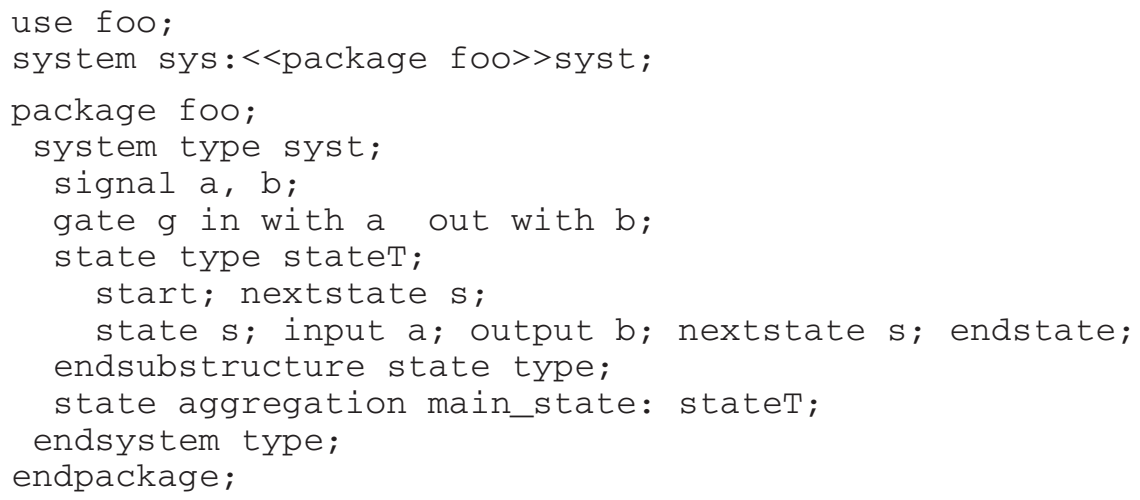

as input, the resulting trace would read

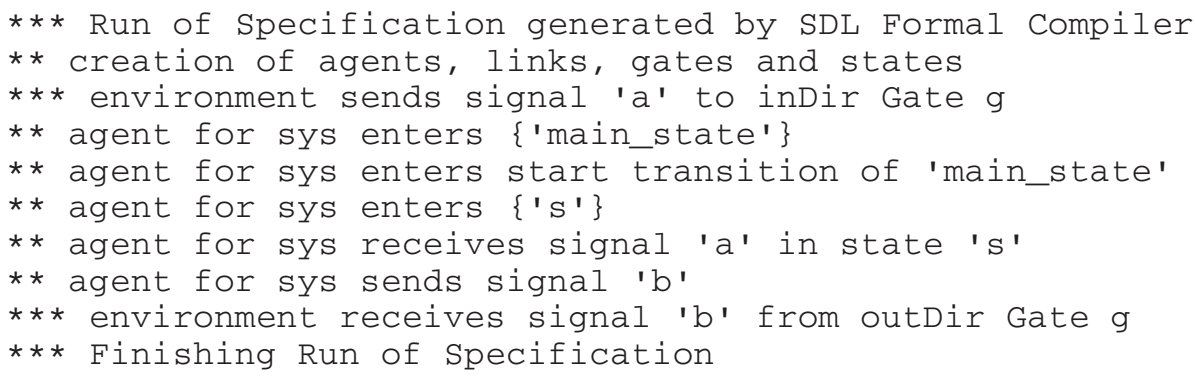

\section{Correcting Errors}

Each time the tools showed unexpected results, an analysis was necessary as to what precisely the problem was. In some cases, we found that simply the expectations were wrong, and that the observed behaviour is really a possible interpretation of the SDL system. In other cases, we traced the error to a bug in the tool chain. If the problem turned out to be an error in the formal semantics, this error was corrected. For this latter category, we counted all errors we corrected, and classified them further. The result of this classification is shown in table 1 below.

It should be noted that the large amount of errors is due to the fact that all errors encountered were counted, even if it was just one missing bracket or a missing parameter. Most of these errors were repaired with little effort.

In [15] it is shown how the tool chain can be used to check and improve the formal semantics definition and how to detect errors in the formal semantics.

\section{Engineering Other Formal Language Definitions}

While we developed the formal semantics and tool support only of a single programming language, SDL-2000, we believe that our approach is directly applicable to 
other programming languages. In order to apply our techniques, the four aspects of a formal semantics (lexis, syntax, static and dynamic semantics) need to be identified. It might be possible to extend the techniques to cover other aspects of language semantics (like non-functional aspects), but we have not studied such extensions.

Table 1. Errors found in the formal semantics

\begin{tabular}{|l|r|r|}
\hline \multicolumn{1}{|c|}{ Category } & Errors in F.2 & \multicolumn{1}{|c|}{ Errors in F.3 } \\
\hline Syntax errors & 352 & 189 \\
\hline Type errors & 88 & 314 \\
\hline Spelling Errors & 115 & 80 \\
\hline Missing or superfluous parameters & 159 & 34 \\
\hline Minor semantic errors & 57 & 227 \\
\hline Incorrect usage of abstract syntax & 144 & 83 \\
\hline New auxiliary functions & & 32 \\
\hline Problems with the abstract syntax 0 & 4 & 3 \\
\hline Major semantic errors & 12 & \\
\hline
\end{tabular}

For lexis and syntax, the major requirement is that the language has a textual notation, and that EBNF can be used to define a super-language (i.e. a grammar that produces a superset of all well-formed programs). Care should be taken to find a "small" super-language, as all programs which are syntactically correct but still ill-formed need to be detected by a well-formedness condition.

Many languages with a textual notation include short-hand notations. For example, in Java, not specifying a base class is equivalent to giving java.lang.Object as the base class. Defining transformation rules is a powerful technique for capturing the semantics of shorthand notations, and should be used wherever possible.

In our experience, using the same grammar on several abstraction levels (i.e. to represent programs with and without shorthands) causes no problems. Wellformedness conditions are best applied to the more abstract program representation, as less special cases have to be considered. "Early" checking of well-formedness conditions should only be applied were mandated by the informal language definition or to safeguard transformation rules.

To express the dynamic semantics, Abstract State Machines have been demonstrated to support various programming language features. However, in the compilation-based approach that we have used, it is more concise to transform the program into primitives of an abstract machine, and to use ASM programs then to give a meaning to such primitives.

Regardless of what formalisation techniques are used, it is essential to accompany them with tools that validate and execute the formal language definition. If a formal language definition uses the same notations as the SDL-2000 formal definition, the tools that we have developed can likely be used with little or no modification.

\section{Comparison with Other Approaches}

For the comparison of the SDL formal semantics with other formal semantics definitions, see e.g. [6]. In the scope of this paper, we only want to have a look at the executable formal semantics works and compiler construction tools. 
It is not a completely new idea to define a formal semantics for a language. There were several other attempts to define programming language semantics formally. However, the direction for the formal semantics was different most of the time. The formal semantics started from the abstract grammar, and was hence used to prove certain properties of the language (good ones and bad ones). It was not really possible to derive a real compiler from the formal language description, because the front-end properties of the language, i.e. the compiler-related part, was not formalised. In addition, many of these attempts to formalise a language only targeted a subset of the language. For example, attempts to provide a formal semantics for the $\mathrm{C}$ and $\mathrm{C}++$ programming languages ([13], [14]) ignore (among other things) the semantics of the pre-processor.

There have also been lots of attempts to define tools that generate compilers including the semantics of the languages, e.g. kimwitu [11], Eli (Paderborn) [12] and other compiler generation tools. It is possible to generate a compiler this way, however, the semantics of the formal texts used to derive the semantics of the language is not really given in a strict mathematical sense.

\section{Summary and Conclusions}

Defining a formal semantics for a real-world language (such as SDL-2000) is a tedious and error-prone work. The advantages of developing such a formal definition (avoidance of ambiguity in the language definition, ability to proof properties of systems) can only materialise if the formal definition itself is nearly free of errors, and procedures are in place to correct errors that have been found.

In our experience, designing and formulating a formal language definition are only a small part of making it actually useful. It needs to be accompanied with a tool chain that helps to validate the formal definition in itself, and gives practitioners of the language a tool to access the formal definition more conveniently.

\section{Acknowledgements}

We would like to thank the SDL Forum Society and Microsoft Research for funding this research, and providing access to both the SDL standard, and beta versions of the AsmL compiler.

\section{References}

1. A. Prinz. Formal Semantics for SDL. Definition and Implementation. Humboldt-Universität zu Berlin, 2001.

2. International Telecommunication Union (ITU). Specification and Description Language (SDL). ITU-T Recommendation Z.100. Geneva, 2000.

3. International Telecommunication Union (ITU). SDL Formal Definition. ITU-T Recommendation Z.100.F, Geneva, 2000.

4. Michael Piefel. Kimwitu++. http://site.informatik.hu-berlin.de/kimwitu++. 
5. Y. Gurevich. Evolving Algebras 1993: Lipari Guide. In E. Börger, editor, Specification and Validation Methods, pages 9-36, Oxford University Press, 1995

6. Eschbach, R., Glässer, U., Gotzhein, R., von Löwis, M., Prinz, A.: Formal Definition of SDL-2000: Compiling and Running SDL Specifications as ASM Models, Journal of Universal Computer Science 7 (11), 2001, Springer, pp. 1025-1050

7. Microsoft Research. AsmL. http://research.microsoft.com/foundations/AsmL/default.html

8. M. v. Löwis, M. Piefel. The Term Processor Kimwitu++. In N. Callaos, T. Leng, B. Sanchez: Proceedings of the $6^{\text {th }}$ World Conference on Systemics, Cybernetics, and Informatics, Orlando, 2002

9. Microsoft Corporation. Microsoft@ Office 2000 Resource Kit. Microsoft Press, 1999

10.T. Archer. Inside C\#. Microsoft Press, 2001

11.P. van Eijk. Tools for LOTOS, a Lotosphere overview. In tutorial proceedings of 11 th Symposium on Protocol Specification, Testing and Verification, Sydney, 1991.

12.U. Kastens. Executable Specifications for Language Implementation. In Fifth International Symposium on Programming Language Implementations and Logic Programming, Tallinn, 1993, Springer LNCS 714, pp. 1-11.

13.Y. Gurevich, J. K. Huggins. The Semantics of the C Programming Language. Springer LNCS 702, Springer, 1993.

14.C. Wallace. The Semantics of the C++ Programming Language. In E. Börger, Specification and Validation Methods, Oxford University Press, 1995.

15.A. Prinz, M. v. Löwis: Generating A Compiler for SDL From The Formal Language Definition in R. Reed (Ed.): SDL 2003: System Design, Proceedings of the 11th International SDL Forum 2003, LNCS 2708, Springer, 2003. 